\title{
Fast Basins and Branched Fractal Manifolds of Attractors of Iterated Function Systems
}

\author{
Michael F. BARNSLEY $Y^{\dagger}$ and Andrew VINCE ${ }^{\ddagger}$ \\ $\dagger$ Mathematical Sciences Institute, Australian National University, Australia \\ E-mail: michael.barnsley@anu.edu.au \\ URL: http://www. superfractals.com \\ $\ddagger$ Department of Mathematics, Univesity of Florida, USA \\ E-mail: avince@ufl.edu
}

Received June 23, 2015, in final form October 13, 2015; Published online October 16, 2015

http://dx.doi.org/10.3842/SIGMA.2015.084

\begin{abstract}
The fast basin of an attractor of an iterated function system (IFS) is the set of points in the domain of the IFS whose orbits under the associated semigroup intersect the attractor. Fast basins can have non-integer dimension and comprise a class of deterministic fractal sets. The relationship between the basin and the fast basin of a point-fibred attractor is analyzed. To better understand the topology and geometry of fast basins, and because of analogies with analytic continuation, branched fractal manifolds are introduced. A branched fractal manifold is a metric space constructed from the extended code space of a point-fibred attractor, by identifying some addresses. Typically, a branched fractal manifold is a union of a nondenumerable collection of nonhomeomorphic objects, isometric copies of generalized fractal blowups of the attractor.
\end{abstract}

Key words: iterated function system; fast basins; fractal continuation; fractal manifold

2010 Mathematics Subject Classification: 05B45; 37B50; 52B50

\section{Introduction}

This paper on iterated function systems concerns related concepts: the fast basin, fractal continuation, an extended code space, and the branched fractal manifold.

The fast basin of an attractor of an iterated function system (IFS) is the set of points, each of which possesses a chaos game orbit [4] that reaches the attractor in finitely many steps. More precisely, let $A$ be an attractor of an IFS $\mathcal{F}$, here defined to be a finite set of homeomorphisms $f_{n}: \mathbb{X} \rightarrow \mathbb{X}, n=1,2, \ldots, N$, mapping a complete metric space $\mathbb{X}$ onto itself. If $f, g \in \mathcal{F}$, then $f \circ g$ denotes the composition of $f$ with $g$. The fast basin of $A$ is the set of points $x \in \mathbb{X}$ such that there exists a finite sequence of digits $\left\{\iota_{n}\right\}_{n=1}^{k} \subset\{1,2, \ldots, N\}^{k}$ with $f_{\iota_{1}} \circ f_{\iota_{2}} \circ \cdots \circ f_{\iota_{k}}(x) \in A$. Equivalently, the fast basin is the complement of the set of points, all of whose orbits do not intersect the attractor. If the maps that comprise the IFS are contractive similitudes on $\mathbb{R}^{n}$, then the fast basin is the union of fractal blowups, as defined by Strichartz [14], of $A$ with respect to the IFS. In general, a fast basin is a union of fractal continuations, generalizing the main idea in [6], of $A$ with respect to the IFS.

Some examples of fast basins, corresponding to geometrically simple IFSs, are presented in Section 3, illustrating that they can have interesting geometrical structure and that they are not easy to understand. Fast basins of affine IFSs may have non-integer Hausdorff dimension, as proved in [2], and thus comprise a class of deterministic fractals which may be suitable for geometrical modelling of real-world objects, extending the reach of ideas initiated by Mandelbrot; see for example the many modelling papers that cite [13] and use IFS theory. But our interest here is in mathematical structure of fast basins, which is relevant to fractal transformations [1], 
tiling theory [8], non-commutative geometry [11], and to a generalization of analytic continuation (see below).

A branched fractal manifold is a certain metric space built from the code space of a pointfibred attractor of an IFS. Its projection onto $\mathbb{X}$ is the fast basin of the attractor. An initial motivation for defining and analyzing branched fractal manifolds was a desire to understand the topological structures of fast basins, at least in the case of simple IFSs consisting of a few affine maps.

A strong motivation for interest in fast basins and branched fractal manifolds is a new view of analytic continuation, introduced in [6]. According to this view, fractal continuation provides an extension of the notion of analytic continuation. As a simple example, consider the IFS defined by the pair of Möbius transformations,

$$
\left(\begin{array}{cc}
-31+4 i & 8+22 i \\
2+11 i & 2-4 i
\end{array}\right) \quad \text { and } \quad\left(\begin{array}{cc}
-25-13 i & -17+14 i \\
-11+7 i & -4+13 i
\end{array}\right)
$$

acting on the Riemann sphere. This IFS has a unique attractor, an arc of a circle (of radius one half, centered at $3 i / 2$ ). The fast basin is the whole circle, i.e., the analytic continuation of the arc. This illustrates a more general situation. In [6] it is proved, under conditions that are not stated here, that the graph of a real analytic function over an interval, say $[0,1]$, is the attractor of an IFS whose maps are analytic on a neighborhood of the graph, and generalized blowups of the graph, generated using the IFS, coincide with the graph of any analytic continuation of the original function, where both are defined. (In [6] and here, we refer to generalized fractal blowups of the graphs of fractal interpolation functions as (fractal) continuations.) In [6] it is further established that all except two fractal continuations of the graphs of any affine fractal interpolation function $g:[0,1] \rightarrow \mathbb{R}$ are defined for all real $x$. Although a fractal continuation coincides with the real analytic continuation of $g$ when $g$ is real analytic on a neighborhood of $[0,1]$, in general the graphs of different continuations of an affine fractal interpolation function do not coincide, except over [0,1]. Instead, their union, which is an example of a fast basin, may be interpreted as an analog or generalization of analytic continuation applied to a fractal function $g$. In this interpretation, different continuations of $g$ belong to different branches, thus the need to understand how these branches fit together and the motivation for the fast basin and branched fractal manifold.

The organization of the paper is as follows. In Section 2 we define point-fibred IFSs, their attractors, duals, basins, fast basins and fractal continuations. A fractal continuation is a generalization of a fractal blowup as defined by Strichartz [14]. Proposition 2.7 states that the fast basin of an attractor is the union of its fractal continuations. Section 3 provides examples of fast basins.

The relationship between the basin and the fast basin of an attractor is the subject of Section 4. Theorem 4.2 shows that the image of a disjunctive point in code space under the coding map (see below) belongs to the boundary of an attractor if and only if the interior of the attractor is empty. Theorem 4.5 relates addresses of points in attractors to whether or not associated continuations contain the basin, using the concept of reversible addresses. As a consequence, Corollary 4.6 states that the fast basin contains the basin if and only if the attractor has nonempty interior.

In Section 5, two symbolic IFSs $Z$ and $\widehat{Z}$ are introduced. The first, $Z=\left\{\mathbb{I}_{+} ; \sigma_{n}, n=\right.$ $1,2, \ldots, N\}$, involves shift maps on $\mathbb{I}_{+}=\{1,2, \ldots, N\}^{\infty}$ and is well known, when $\mathcal{F}$ is contractive, to be related to the attractor $A$ by the following commutative diagram

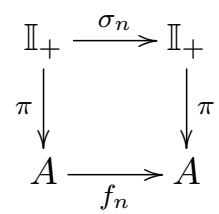


This involves the coding map or addressing function, a continuous surjection $\pi: \mathbb{I}_{+} \rightarrow A$. However, $Z$ is not an IFS, as defined in this paper, as the maps of $Z$ are not invertible and its fast basin is undefined. This is remedied by the introduction of $\widehat{Z}=\left\{\mathbb{I} ; \sigma_{n}, n=\right.$ $1,2, \ldots, N\}$ whose maps are invertible. Here $\mathbb{I}$ is a certain shift invariant subspace of $\mathbb{I}_{0}=$ $\{-N, \ldots,-1,1,2, \ldots, N\}^{\infty}$. The attractor of $\widehat{Z}$ is $\mathbb{I}_{+}$and its fast basin, $\widehat{\mathbb{I}} \subset \mathbb{I}$, provides a symbolic representation for any attractor of $\mathcal{F}$ and its fast basin. It is shown that the coding map $\pi: \mathbb{I}_{+} \rightarrow A$ can be extended to various subspaces of $\mathbb{I}$, including $\widehat{\mathbb{I}}$, and that the action of shift maps on these subspaces is (semi-)conjugate to the action of the $f_{n} \mathrm{~s}$ (and/or their inverses) on corresponding subspaces of $\mathbb{X}$, including the fast basin of $A$. Dual relationships also hold in certain cases. These results are summarized by the commutative diagrams in Theorem 5.6 and Corollaries 5.8 and 5.9 .

The branched fractal manifold $\mathbb{L}$ associated with a point-fibred attractor of an invertible IFS is defined in Section 6. It is a metric space constructed from the symbolic fast basin $\widehat{\mathbb{I}}$ by identifying some addresses. It is a disjoint union of certain leaves, each leaf being homeomorphic to one of at most $N+1$ subsets of $A$, but $\mathbb{L}$ itself may not be locally compact. Also, $\mathbb{L}$ contains isometric copies, called sheets, of all the fractal continuations of the original attractor. The branched fractal manifold contains, in a concise way, information on the global addressing discussed in Section 5. A description of $\mathbb{L}$, providing its main properties, is the content of Theorems 6.4 and 6.5. Examples are provided.

Relationships between this paper and related work in the literature are discussed in Section 7.

\section{IFSs attractors, their basins, fast basins, and continuations}

Let $\mathbb{N}=\{1,2,3, \ldots\}$ and $\mathbb{N}_{0}=\{0,1,2, \ldots\}$. Throughout this paper an iterated function system $(I F S)$ is a complete metric space $\mathbb{X}$ together with a finite set of homeomorphisms $f_{n}: \mathbb{X} \rightarrow \mathbb{X}$, $n=1,2, \ldots, N$, and is denoted by

$$
\mathcal{F}=\mathcal{F}_{\mathbb{X}}=\left\{\mathbb{X} ; f_{1}, f_{2}, \ldots, f_{N}\right\}
$$

We use the same symbol $\mathcal{F}$ for the IFS and for the set of functions $\left\{f_{1}, f_{2}, \ldots, f_{N}\right\}$.

Let $H=H_{\mathbb{X}}$ be the collection of nonempty compact subsets of $\mathbb{X}$ and define $F: H \rightarrow H$ by

$$
F(C)=\bigcup_{f \in \mathcal{F}} f(C)
$$

for all $C \in H$, where $f(C)=\{f(x): x \in C\}$. We extend naturally $F$ to a map on the collection of all subsets of $\mathbb{X}$. For $S \subset \mathbb{X}$, define $F^{0}(S)=S$ and let $F^{k}(S)$ denote the $k$-fold composition of $F$ applied to $S$, namely, the union of $\left\{\iota_{n}\right\}_{n=1}^{k} \subset\{1,2, \ldots, N\}^{k}$ with $f_{\iota_{1}} \circ f_{\iota_{2}} \circ \cdots \circ f_{\iota_{k}}(S)$ over all finite words $\iota_{1} \iota_{2} \cdots \iota_{k}$ of length $k$.

Let $d=d_{\mathbb{X}}$ be the metric on $\mathbb{X}$, and let $d_{H}=d_{H_{\mathbb{X}}}$ be the corresponding Hausdorff metric on $H_{\mathbb{X}}$. Throughout, the topology on $H_{\mathbb{X}}$ is the one induced by $d_{H_{\mathbb{X}}}$. It is well known that $\left(H, d_{H}\right)$ is a complete metric space because $(\mathbb{X}, d)$ is complete, and that if $(\mathbb{X}, d)$ is compact then $\left(H, d_{H}\right)$ is compact.

Definition 2.1. An attractor of the IFS $\mathcal{F}$ is a set $A \in H$ such that

1) $F(A)=A$, and

2) there is an open set $U \subset \mathbb{X}$ such that $A \subset U$ and $\lim _{k \rightarrow \infty} F^{k}(C)=A$, for all $S \in H$ with $C \subset U$, where the limit is w.r.t. the metric $d_{H}$. 
The union of all open sets $U$, such that statement 2 of Definition 2.1 is true, is called the basin of the attractor $A$ (w.r.t. $\mathcal{F}$ ). If $B=B(A)$ denotes the basin of $A$, then it can be proved that statement 2 of Definition 2.1 holds with $U$ replaced by $B$. That is, the basin of the attractor $A$ is the largest open set $U$ such that statement 2 of Definition 2.1 holds. An IFS may not possess an attractor, or it may possess multiple attractors. Examples and further discussion can be found in [5] and in references therein.

The IFS $\mathcal{F}$ is said to be contractive if there is a $\lambda \in[0,1)$ such that

$$
d_{\mathbb{X}}(f(x), f(y)) \leq \lambda d_{\mathbb{X}}(x, y)
$$

for all $f \in \mathcal{F}$ and all $x, y \in \mathbb{X}$. A basic result of [10] is that a contractive IFS possesses a unique attractor $A$, with $B(A)=\mathbb{X}$.

Note that, in this paper, each $f_{n}: \mathbb{X} \rightarrow \mathbb{X}$ is a homeomorphism and so has a continuous inverse $f_{n}^{-1}: \mathbb{X} \rightarrow \mathbb{X}$ which is also a homeomorphism. This allows us to define an "inverse" of the IFS $\mathcal{F}$ as follows.

Definition 2.2. The dual IFS is

$$
\mathcal{F}^{*}:=\left\{\mathbb{X} ; f_{n}^{-1}, n=1,2, \ldots, N\right\} .
$$

If $A$ is an attractor of the IFS $\mathcal{F}$, then the set $A^{*}:=\mathbb{X} \backslash B(A)$ is called the dual repeller of $A$ (w.r.t. $\mathcal{F}$ ). The dual repeller $A^{*}$, under certain conditions [7], is an attractor of the dual IFS $\mathcal{F}^{*}$. This can occur, for example, when $\mathcal{F}_{\widehat{\mathbb{C}}}$ is a Möbius IFS on the Riemann sphere $\widehat{\mathbb{C}}$ [17].

For an infinite word $\iota=\iota_{1} \iota_{2} \iota_{3} \cdots, \iota_{i} \in\{1,2, \ldots, N\}$, let

$$
\iota \mid k=\iota_{1} \iota_{2} \iota_{3} \cdots \iota_{k}, \quad f_{\iota \mid k}=f_{\iota_{1}} \circ f_{\iota_{2}} \circ \cdots \circ f_{\iota_{k}},
$$

and $f_{\varnothing}=f_{\iota \mid 0}=i d_{\mathbb{X}}$, the identity map on $\mathbb{X}$.

Definition 2.3. An attractor $A$ of $\mathcal{F}$ is point-fibred (w.r.t. $\mathcal{F}$ ) if

$$
\lim _{k \rightarrow \infty} f_{\iota \mid k}(C) \subset A,
$$

is a singleton subset of $\mathbb{X}$, for all $\iota \in\{1,2, \ldots, N\}^{\infty}$ independent of $C \subset B(A)$ with $C \in H$, where convergence is with respect to the Hausdorff metric.

All attractors in this paper are assumed to be point-fibred. It follows from results in [10] that if the IFS $\mathcal{F}$ is contractive, then its attractor is point-fibred. The point-fibred property allows for an addressing scheme for points of the attractor (Definition 2.4). The following notation will be used throughout this paper. Let $N \in \mathbb{N}$ and let

$$
\begin{array}{ll}
I_{+}=\{+1,+2, \ldots,+N\}, & \mathbb{I}_{+}=I_{+}^{\infty}, \\
I_{-}=\{-1,-2, \ldots,-N\}, & \mathbb{I}_{-}=I_{-}^{\infty}, \\
I=I_{+} \cup I_{-}, & \mathbb{I}_{0}=I^{\infty}, \\
\mathbb{I}=\left\{\iota \in \mathbb{I}_{0}: \iota_{i} \neq-\iota_{i+1} \text { for all } i \in \mathbb{N}\right\} .
\end{array}
$$

Let $d_{\mathbb{I}}$ be the metric on $\mathbb{I}_{0}$ defined by

$$
d_{\mathbb{I}}(\iota, \omega)= \begin{cases}\max \left\{2^{-k}: k \in \mathbb{N}, \iota_{k} \neq \omega_{k}\right\} & \text { if } \iota \neq \omega, \\ 0 & \text { if } \iota=\omega,\end{cases}
$$

for all $\iota, \omega \in \mathbb{I}_{0}$. The metric $d_{\mathbb{I}}$ induces the product topology on $\mathbb{I}_{0}$, and the metric spaces $\left(\mathbb{I}, d_{\mathbb{I}}\right)$, $\left(\mathbb{I}_{+}, d_{\mathbb{I}}\right),\left(\mathbb{I}_{-}, d_{\mathbb{I}}\right)$ are compact subspaces of the compact metric space $\left(\mathbb{I}_{0}, d_{\mathbb{I}}\right)$.

The space $\left(\mathbb{I}_{+}, d_{\mathbb{I}}\right)$ is sometimes called the code space or address space for an attractor of $\mathcal{F}$ because it provides addresses for the points of $A$, as given in the following definition. 
Definition 2.4. Let $A$ be a point-fibred attractor of an IFS $\mathcal{F}$. According to Definition 2.3, there is a map $\pi: \mathbb{I}_{+} \rightarrow A \subset \mathbb{X}$, called the coding map, that is well defined by

$$
\pi(\iota)=\lim _{k \rightarrow \infty} f_{\iota \mid k}(b)
$$

for all $\iota \in \mathbb{I}_{+}$, and the limit is independent of $b \in B(A)$. It is readily proved that $\pi$ is continuous and that $\pi\left(\mathbb{I}_{+}\right)=A$. The set-valued inverse $\pi^{-1}(a)$ comprises the set of addresses of the point $a \in A$ (w.r.t. $\mathcal{F}$ ).

Definition 2.5. The fast basin $\widehat{B}=\widehat{B}(A)$ of an attractor $A$ of an IFS $\mathcal{F}$ is

$$
\widehat{B}=\left\{x \in \mathbb{X}: F^{K}(\{x\}) \cap A \neq \varnothing \text { for some } K \in \mathbb{N}\right\} .
$$

The relationship between the basin $B$ and the fast basin $\widehat{B}$ is complicated because neither $B \subset \widehat{B}$ nor $\widehat{B} \subset B$ holds in general. This topic is discussed in Section 4 .

Fractal continuations were introduced in [6] in the context of fractal interpolation, and in [5] in the context of fractal tiling. (See also the last part of Section 1 for a discussion of related notions.) These situations are generalized in Definition 2.6. Extend the notation of equation (2.1) as follows. For $\iota \in \mathbb{I}_{0}$ define $-\iota$ by

$$
(-\iota)_{k}:=-\iota_{k}
$$

for all $k \in \mathbb{N}$ and

$$
f_{-j}=\left(f_{j}\right)^{-1}
$$

for $j=1,2, \ldots, N$.

Definition 2.6. Let $A$ be an attractor of an IFS $\mathcal{F}=\left\{\mathbb{X} ; f_{n}, n \in I_{+}\right\}$. For $\theta \in \mathbb{I}_{+}$, the continuation $B_{\theta}$ of $A$ (w.r.t. $\mathcal{F}$ ) is defined to be

$$
B_{\theta}=\bigcup_{k \in \mathbb{N}} f_{-\theta \mid k}(A)=A \cup \bigcup_{k \in \mathbb{N}} f_{\theta_{1}}^{-1} \circ f_{\theta_{2}}^{-1} \circ \cdots \circ f_{\theta_{k}}^{-1}(A) .
$$

Note that this is a nested union in the sense that $f_{-\theta \mid k}(A) \subset f_{-\theta \mid k+1}(A)$.

The set $B_{\theta}$ is referred to as a continuation of $A$ or a fractal continuation of $A$. The family $\left\{B_{\theta}: \theta \in \mathbb{I}_{+}\right\}$is referred to as the set of continuations of $A$. We also write $B_{\theta \mid k}=f_{-\theta \mid k}(A)$, which is referred to as a finite continuation of A. Clearly

$$
B_{\theta}=\bigcup_{k \in \mathbb{N}_{0}} B_{\theta \mid k}
$$

The paper [6] concerns the special case of fractal continuations of fractal functions. In that case it is proved, under special conditions, the most important of which is that the IFS consists of analytic functions, that the set of continuations is uniquely determined by the attractor, independent of the analytic IFS used to generate the attractor.

The following result, stating that the fast basin is the union of all the continuations, is readily verified.

Proposition 2.7. Let $A$ be an attractor of an IFS $\mathcal{F}=\left\{\mathbb{X} ; f_{n}, n \in I_{+}\right\}$. If $\widehat{B}$ is the fast basin of $A$ and $\left\{B_{\theta}: \theta \in \mathbb{I}_{+}\right\}$is the set of continuations of $A$, then

$$
\widehat{B}=\bigcup_{\theta \in \mathbb{I}_{+}} B_{\theta} .
$$




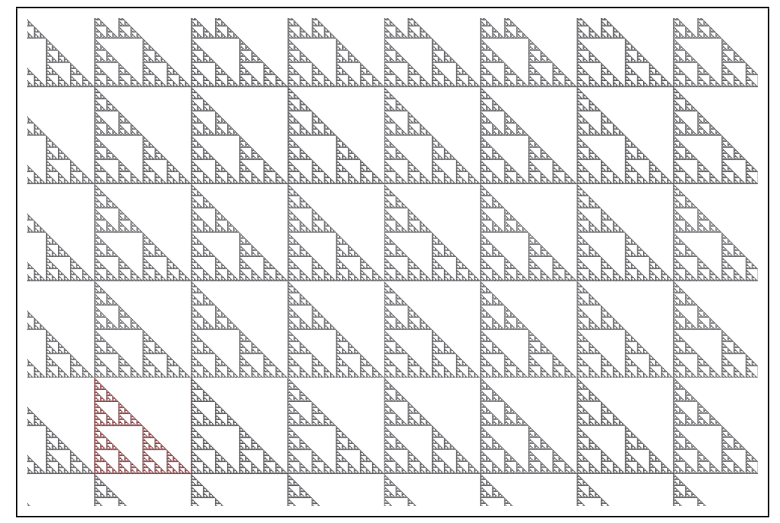

Figure 1. This illustrates part of the fast basin of the attractor of the iterated function system $\mathcal{F}=$ $\left\{\mathbb{R}^{2} ;(x / 2, y / 2),((x+1) / 2, y / 2),(x / 2,(y+1) / 2)\right\}$. The attractor is rendered in red at the lower left.

\section{Fast basin examples}

Example 3.1 (Cantor set). Routine calculation shows that the fast basin of the standard Cantor set $\mathcal{C} \subset \mathbb{R}$, w.r.t. the IFS $\{\mathbb{R} ; x / 3, x / 3+2 / 3\}$, is

$$
\widehat{B}=\bigcup_{k \in \mathbb{Z}}(\mathcal{C}+2 k)
$$

We use the notation for Minkowski sum, $(\mathcal{C}+2 k):=\{x+2 k: x \in \mathcal{C}\}$.

Example 3.2 (Sierpinski gasket). The fast basin of a Sierpinski triangle $\triangle \subset \mathbb{R}^{2}$, with vertices at $v_{1}, v_{2}, v_{3} \in \mathbb{R}^{2}$, w.r.t. the IFS $\left\{\mathbb{R}^{2} ;\left(x+v_{1}\right) / 2,\left(x+v_{2}\right) / 2,\left(x+v_{3}\right) / 2\right\}$, is $\widehat{B}=\cup_{t \in \mathcal{G}}(\triangle+t)$ where $\mathcal{G}$ is the group generated by the set of translations by $\left\{v_{1}-v_{2}, v_{2}-v_{3}, v_{3}-v_{1}\right\}$. This may be proved by induction, using $A=\cup_{i} f_{i}(A)$ and $f_{v_{i}}^{-1} \circ f_{v_{j}}$ is translation by $v_{j}-v_{i}$. Fig. 1 shows $\widehat{B}$ when $v_{1}=(0,0), v_{2}=(1,0)$, and $v_{3}=(0,1)$.

Example 3.3 (other affine IFSs). Fig. 2 illustrates part of the fast-basin of a contractive affine IFS $\left\{\mathbb{R}^{2} ; f_{1}, f_{2}\right\}$, where

$$
f_{1}(x, y)=\left(\frac{x}{2}+\frac{y}{2 \sqrt{3}}-1, \frac{x}{2 \sqrt{3}}-\frac{y}{2}\right), \quad f_{2}(x, y)=\left(\frac{x}{2}-\frac{y}{2 \sqrt{3}}+1,-\frac{x}{2 \sqrt{3}}-\frac{y}{2}\right) .
$$

The attractor is the segment of the Koch snowflake curve near the center of the figure. The fast basin is the union of the boundaries of the tiles of a tiling of the plane by Koch snowflakes and other related tiles.

Fig. 3 illustrates part of the fast basin for the contractive affine IFS $\left\{\mathbb{R}^{2} ; f_{1}, f_{2}\right\}$, where

$$
f_{1}(x, y)=\left(\frac{x}{2}+\frac{1}{2}, \frac{x}{2}+\frac{2 y}{5}+\frac{1}{4}\right), \quad f_{2}(x, y)=\left(\frac{x}{2}-\frac{1}{2},-\frac{x}{2}+\frac{2 y}{5}+\frac{1}{4}\right),
$$

whose attractor, at the center of the image, is the graph of a fractal interpolation function. At each branch point, there is a countable infinity of distinct branches.

Both figures in this example were obtained by direct computation.

Example 3.4 (topological dimension of the fast basin). We use the following definition: an orbit of a point $x \in \mathbb{X}$, under the IFS $\mathcal{F}=\left\{\mathbb{X} ; f_{1}, \ldots, f_{N}\right\}$, is $\left\{f_{\iota_{1}} \circ f_{\iota_{2}} \circ \cdots \circ f_{\iota_{k}}(x)\right\}_{k=1}^{\infty} \subset \mathbb{X}$ for some $\iota \in\{1,2, \ldots, N\}^{\infty}$. If $\mathcal{F}$ is an IFS on $\mathbb{R}^{M}$ consisting of affine functions and whose attractor $A$ is contained in a proper linear subspace $V$ of $\mathbb{R}^{M}$, then it is easy to show that the 


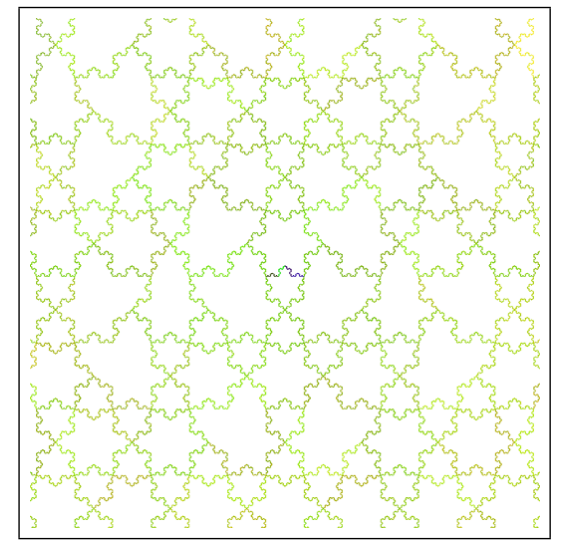

Figure 2. See Example 3.3. This shows the fast basin for the Koch curve using an IFS of similitudes. The Koch curve is located near the center, in various colours.

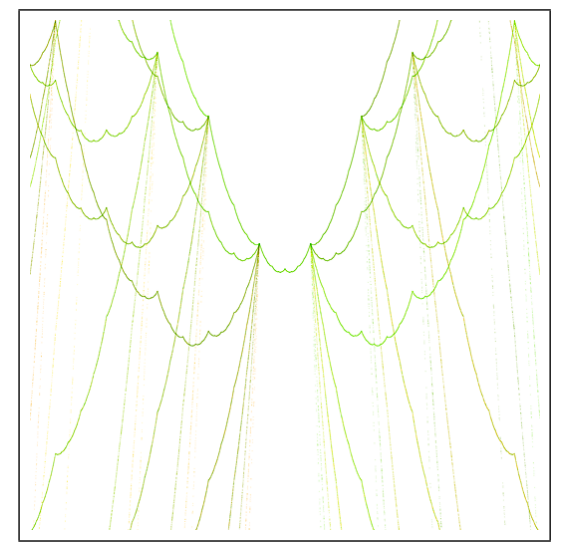

Figure 3. See Example 3.3.

fast basin of $A$ is also contained in $V$. Moreover, any orbit of any point not in $V$ has empty intersection with $A$. It follows that the topological dimension of the fast basin of $A$ is strictly less than that of the underlying space. For instance, if $M=N=2$, and

$$
f_{1}(x, y)=(x / 2, y / 2), \quad f_{2}(x, y)=((x+1) / 2, y / 2)
$$

for all $(x, y) \in \mathbb{R}^{2}$, then $A=[0,1] \times\{0\} \subset \mathbb{R} \times\{0\}, B=\mathbb{R}^{2}$, and $\widehat{B} \subset \mathbb{R} \times\{0\}$. Any orbit of any point in $\mathbb{R}^{2} \backslash\{(x, 0): x \in \mathbb{R}\}$ does not meet the attractor (although the closure of the orbit does meet the attractor) while, for any point in $\mathbb{R}$, there exists an orbit that reaches $A$ in finitely many steps, i.e., the orbit intersects the attractor.

Example 3.5. An example, illustrating a relationship between a fast basin and analytic continuation, is provided by the contractive IFS

$$
\mathcal{F}=\left\{\mathbb{C}^{2} ; f_{+1+i}, f_{-1+i}, f_{+1-i}, f_{-1-i}\right\},
$$

where

$$
\begin{aligned}
& f_{ \pm 1-i}\left(\begin{array}{c}
z \\
w
\end{array}\right)=\left(\begin{array}{cc}
\frac{1}{2} & 0 \\
\frac{ \pm 1-i}{2} & \frac{1}{4}
\end{array}\right)\left(\begin{array}{c}
z \\
w
\end{array}\right)+\left(\begin{array}{c}
\frac{ \pm 1-i}{2} \\
\frac{ \pm i}{2}
\end{array}\right), \\
& f_{ \pm 1+i}\left(\begin{array}{c}
z \\
w
\end{array}\right)=\left(\begin{array}{ll}
\frac{1}{2} & 0 \\
\frac{ \pm 1+i}{2} & \frac{1}{4}
\end{array}\right)\left(\begin{array}{c}
z \\
w
\end{array}\right)+\left(\begin{array}{c}
\frac{ \pm 1+i}{2} \\
\frac{ \pm i}{2}
\end{array}\right) .
\end{aligned}
$$


By following arguments similar to ones in [6], we find that the unique attractor $A$ of $\mathcal{F}$ is the graph of $z \mapsto z^{2}$ over the square $-1 \leq \operatorname{Re} z, \operatorname{Im} z \leq+1$. We also find that the fast basin $\widehat{B}$ of $A$ (w.r.t. $\mathcal{F}$ ) is the manifold

$$
\left\{\left(z, z^{2}\right): z \in \mathbb{C}\right\} \text {. }
$$

Example 3.6. An example that is related to a Schottky group is provided by the loxodromic Möbius IFS

$$
\mathcal{F}=\left\{\widehat{\mathbb{C}} ; f_{1}(z)=\frac{a z-i(2+\sqrt{3})}{z-i(2+\sqrt{3})+a-1}, f_{2}(z)=\frac{a z+i(2+\sqrt{3})}{-z-i(2+\sqrt{3})+a-1}\right\}
$$

where $a=\frac{-i(2+\sqrt{3}) C+1}{1-C}, C \in \mathbb{C}$ with $|C| \notin\{0,1\}, f_{1}(1)=1$ and $f_{2}(-1)=-1$. We find

$$
f_{1}^{\prime}(1)=f_{2}^{\prime}(-1)=C
$$

so if $|C|$ is sufficiently small, then $\mathcal{F}$ has a totally disconnected attractor $A$, located within two small circles, one centered at +1 and one centered at -1 . This is because the center of each circle is an attractive fixed point of one of the maps, and the derivative of each map, at its attractive fixed point, has magnitude $|C|$. Similarly, in this case, the dual repeller $A^{*}$ is also totally disconnected and is located within two small circles, one centered at $(2+\sqrt{3}) i$ and the other at $-(2+\sqrt{3}) i$. The maps $f_{1}, f_{2}$ are the generators of a Schottky group whose limit set $\Lambda$ is also totally disconnected, and we have $A \subset \Lambda, A^{*} \subset \Lambda$, and $\widehat{B} \subset \Lambda$. It follows that $\widehat{B}$ is totally disconnected.

\section{When does the fast basin contain the basin?}

Consider the following examples showing that, in general, there is no containment relationship between the basin and the fast basin. Since the IFS

$$
\left\{\mathbb{R} ; \frac{1}{2} x, \frac{1}{2} x+\frac{1}{2}\right\}
$$

is contractive, the basin $B=\mathbb{R}$. It is not hard to show, for example via Proposition 2.7, that the fast basin $\widehat{B}=\mathbb{R}$. Therefore, $\widehat{B}=B$ for this IFS. Likewise, the IFS

$$
\left\{\mathbb{R} ; \frac{1}{3} x, \frac{1}{3} x+\frac{2}{3}\right\}
$$

is contractive, but, as in Example (3.1), the fast basin is not all of $\mathbb{R}$. Therefore $\widehat{B} \subsetneq B$ for this IFS. Finally consider the IFS $\mathcal{F}=\left\{\mathbb{P}^{1} ; f_{1}, f_{2}\right\}$, where $\mathbb{P}^{1}$ is 1 -dimensional projective space, homeomorphic to the circle, and which we denote by $\mathbb{R} \cup\{\infty\}$ (the reals with an additional point at infinity). The functions are

$$
f_{1}(x)=\frac{9 x}{20-2 x}, \quad f_{2}(x)=\frac{11 x+9}{2 x+18} .
$$

The attractor $A$ of $\mathcal{F}$ is the interval $[0,1]$; the attractor $A^{*}$ of the dual IFS is $\mathbb{R} \backslash\{-9 / 2,11 / 2\}$. Therefore the basin of $A$ is $B=\mathbb{R} \backslash A^{*}=\{-9 / 2,11 / 2\}$. It is straightforward to check that the fast basin is $\widehat{B}=\mathbb{R}$. Therefore, in this example, $B \subsetneq \widehat{B}$.

From the above examples we see that all three possibilities $\widehat{B}=B, \widehat{B} \subsetneq B, B \subsetneq \widehat{B}$ can occur. However, if the attractor $A$ has nonempty interior (in the topology of $\mathbb{X}$ ), then $B \subseteq \widehat{B}$ (see Corollary 4.6). 
Define the shift map $S: \mathbb{I}_{0} \rightarrow \mathbb{I}_{0}$ by

$$
S\left(\iota_{1} \iota_{2} \cdots\right)=\iota_{2} \iota_{3} \cdots
$$

for all $\iota \in \mathbb{I}_{0}$. Note that $S$ is continuous and maps $\mathbb{I}$ onto $\mathbb{I}, \mathbb{I}_{+}$onto $\mathbb{I}_{+}$, and $\mathbb{I}_{-}$onto $\mathbb{I}_{-}$.

Definition 4.1. A word $\theta \in \mathbb{I}_{+}$is disjunctive if every finite word is a subword of $\theta$. In fact, if $\theta$ is disjunctive, then every finite word (in the alphabet $I_{+}$) appears as a subword in $\iota$ infinitely many times. Moreover, an equivalent definition of a disjunctive word is that its orbit under the shift map is dense in the code space. Denote the interior of set $S$ by $S^{\circ}$ and the closure by $\bar{S}$.

Theorem 4.2. Let $A$ be an attractor of an IFS $\mathcal{F}$, and let $\theta \in \mathbb{I}_{+}$be disjunctive. Then $\pi(\theta) \in A^{\circ}$ if and only if $A^{\circ} \neq \varnothing$.

Proof. Since $\theta \in \mathbb{I}_{+}$is disjunctive, the orbit, $\left\{S^{k}(\theta)\right\}_{k=0}^{\infty}$, of $\theta$ under the shift map $S: \mathbb{I}_{+} \rightarrow \mathbb{I}_{+}$ is dense in $\mathbb{I}_{+}$. Since $\pi$ is continuous, $\left\{\pi_{\mathcal{F}}\left(S^{k}(\theta)\right)\right\}_{k=0}^{\infty}$ is dense in $A$. By way of contradiction assume that $\pi_{\mathcal{F}}(\theta) \in \partial A$, the boundary of $A$. Since each $f \in \mathcal{F}$ is a homeomorphism, $f\left(A^{\circ}\right) \subset A^{\circ}$ or, equivalently, $\left(\left.f\right|_{A}\right)^{-1}(\partial A) \subset \partial A$. Therefore $\pi\left(S^{k}(\theta)\right) \in \partial A$ for all $k \in \mathbb{N}_{0}$, which would mean that $A=\overline{\left\{\pi\left(S^{k}(\theta)\right)\right\}_{k=0}^{\infty}} \subset \overline{\partial A}=\partial A$ which implies $A^{\circ}=\varnothing$, which contradicts $A^{\circ} \neq \varnothing$.

Theorem 4.5 below provides sufficient conditions for a fractal continuation of an attractor to contain the basin of the attractor. It involves the equivalent notions of a full and a reversible word in $\mathbb{I}_{+}$. These concepts were introduced in [8]; the equivalence of full and reversible is part of Theorem 4.4 .

Definition 4.3. If $A$ is an attractor of an IFS $\mathcal{F}$, call $\theta \in \mathbb{I}_{+}$full if there exists a nonempty compact set $A^{\prime} \subset A^{\circ}$ such that, for any positive integer $M$, there exist $n>m \geq M$ such that

$$
f_{\theta_{n}} \circ f_{\theta_{n-1}} \circ \cdots \circ f_{\theta_{m+1}}(A) \subset A^{\prime} .
$$

Call $\theta \in \mathbb{I}_{+}$reversible w.r.t. an attractor $A$ and IFS $\mathcal{F}$ if there exists an $\omega=\omega_{1} \omega_{2} \cdots \in \mathbb{I}_{+}$ such that $\omega$ is the address of some point in $A^{\circ}$ and, for every pair of positive integers $M$ and $L$, there is an integer $m \geq M$ such that

$$
\omega_{1} \omega_{2} \cdots \omega_{L}=\theta_{m+L} \theta_{m+L-1} \cdots \theta_{m+1} .
$$

In some cases it is easy to check if a word is reversible. For example, if $\theta=\theta_{1} \theta_{2} \cdots \theta_{k} \theta_{1} \theta_{2} \cdots$ $\theta_{k} \theta_{1} \cdots$ is periodic and $\pi\left(\theta_{k} \theta_{k-1} \cdots \theta_{1} \theta_{k} \theta_{k-1} \cdots \theta_{1} \theta_{k} \cdots\right)$ lies in the interior of $A$, then $\theta$ is reversible.

Theorem 4.4 ([8, Theorem 3.7]). For an IFS $\mathcal{F}$, let $A$ be an attractor. With respect to $A$ and $\mathcal{F}$ :

1. There are inf initely many disjunctive words in $\mathbb{I}_{+}$for $N \geq 2$.

2. If $A^{\circ} \neq \varnothing$, then every disjunctive word is reversible.

3. A word is reversible if and only if it is full.

Theorem 4.5. Let $A$ be an attractor of an IFS $\mathcal{F}$ with basin $B$. If $\theta \in \mathbb{I}_{+}$is full/reversible, then

$$
B \subset B_{\theta}
$$


Proof. Let $x \in B$. It suffices to show that $x \in f_{\theta_{1}}^{-1} \circ f_{\theta_{2}}^{-1} \circ f_{\theta_{3}}^{-1} \circ \cdots \circ f_{\theta_{n}}^{-1}(A)$ for some $n$, or equivalently

$$
f_{\theta_{n}} \circ f_{\theta_{n-1}} \circ \cdots \circ f_{\theta_{1}}(x) \in A .
$$

By the definition of attractor, for any $\epsilon>0$ there is an $M_{\epsilon}$ such that if $m \geq M_{\epsilon}$, then

$$
F^{m}(x) \subset A_{\epsilon},
$$

where $A_{\epsilon}$ is the open $\epsilon$-neighborhood of $A$. Because $\theta$ is assumed to be full, there exists a compact set $A^{\prime}$ with $A^{\prime} \subset A^{\circ}$ with the property that, for any $M$ there exist $n>m \geq M$ such that

$$
f_{\theta_{n}} \circ \cdots \circ f_{\theta_{m+1}}\left(A^{\prime}\right) \subset A^{\circ} .
$$

This implies that there exists an $\epsilon_{0}$-neighborhood $A_{\epsilon_{0}}$ of $A$ such that

$$
f_{\theta_{n}} \circ \cdots \circ f_{\theta_{m+1}}\left(A_{\epsilon_{0}}\right) \subset A^{\circ}
$$

for some $\epsilon_{0}>0$. If $M \geq M_{\epsilon_{0}}$, then

$$
f_{\theta_{n}} \circ \cdots \circ f_{\theta_{m+1}} \circ f_{\theta_{m}} \circ \cdots \circ f_{\theta_{1}}(x) \in f_{\theta_{n}} \circ \cdots \circ f_{\theta_{m+1}}\left(A_{\epsilon_{0}}\right) \subset A,
$$

as required.

Corollary 4.6. Let $A$ be an attractor of an IFS $\mathcal{F}$ on a complete metric space $\mathbb{X}$, with basin $B$ and fast basin $\widehat{B}$. Then $B \subseteq \widehat{B}$ if and only if $A^{\circ} \neq \varnothing$. Moreover, if $\mathcal{F}$ is contractive and $A^{\circ} \neq \varnothing$, then $B=\widehat{B}=\mathbb{X}$.

Proof. Assume that $A^{\circ} \neq \varnothing$. Let $\theta$ be a disjunctive word; by Theorem 4.4 there are infinitely many. By the same theorem $\theta$ is full and reversible. By Theorem 4.5 and Proposition 2.7 we have $B \subseteq B_{\theta} \subseteq \widehat{B}$. If $\mathcal{F}$ is contractive, then, as mentioned after the definition of contractive in Section 2 (see also [10]), it is well known that $B=\mathbb{X}$.

Conversely, assume that $A^{\circ}=\varnothing$. If $A$ is an attractor, the fast basin of $A$ is

$$
\widehat{B}=\left\{\left(f_{\iota}\right)^{-1}(A): \iota \in \mathbb{I}_{+}^{*}\right\},
$$

where $\mathbb{I}_{+}^{*}$ is the set of all finite sequences in the alphabet $I_{+}$. Since $\left(f_{\iota}\right)^{-1}(A)$ has empty interior, $\widehat{B}$ is nowhere dense by the Baire category theorem. Since $B$ is open, clearly $B \nsubseteq \widehat{B}$.

\section{Symbolic IFSs and the extended coding map}

The goal of this section is to extend the classic coding map given in Definition 2.4. We begin with two examples of "symbolic IFSs". It may be helpful for the reader, at this juncture, to recall the notation introduced around equation $(2.2)$ for $\mathbb{I}_{0}, \mathbb{I}, \mathbb{I}_{+}, \mathbb{I}_{-}, I, I_{+}$and $I_{-}$.

For $n \in I$, define the inverse shift maps $\sigma_{n}: \mathbb{I}_{0} \rightarrow \mathbb{I}_{0}$, by

$$
\sigma_{n}\left(\iota_{1} \iota_{2} \cdots\right)=n \iota_{1} \iota_{2} \cdots,
$$

and define $\sigma_{n}: \mathbb{I} \rightarrow \mathbb{I}$ by

$$
\sigma_{n}\left(\iota_{1} \iota_{2} \cdots\right)= \begin{cases}n \iota_{1} \iota_{2} \cdots & \text { if } \iota_{1} \neq-n \\ \iota_{2} \iota_{3} \cdots & \text { if } \iota_{1}=-n\end{cases}
$$


Example 5.1 (first symbolic IFS). If

$$
Z=\left\{\mathbb{I}_{+} ; \sigma_{n}, n \in I_{+}\right\},
$$

then $Z$ is an IFS each of whose functions is a contraction with scaling factor $1 / 2$. The unique attractor is $\mathbb{I}_{+}$. Not that this example does not strictly fit the definition of an IFS as given in Section 2 because $\sigma_{n}$ is not a homeomorphism as it is not surjective.

Example 5.2 (second symbolic IFS). If

$$
\widehat{Z}=\left\{\mathbb{I} ; \sigma_{n}, n \in I_{+}\right\},
$$

then $\widehat{Z}$ is an IFS, for which it is easily verified that each function $\sigma_{n}: \mathbb{I} \rightarrow \mathbb{I}$ is bi-Lipshitz with

$$
\frac{1}{2} d_{\mathbb{I}}(\iota, \omega) \leq d_{\mathbb{I}}\left(\sigma_{n}(\iota), \sigma_{n}(\omega)\right) \leq 2 d_{\mathbb{I}}(\iota, \omega)
$$

for all $\iota, \omega \in \mathbb{I}$. In particular, each $\sigma_{n}$ is a homeomorphism with inverse $\sigma_{-n}: \mathbb{I} \rightarrow \mathbb{I}$. The dual IFS is

$$
\widehat{Z}^{*}=\left\{\mathbb{I} ; \sigma_{n}, n \in I_{-}\right\} .
$$

Although neither $\widehat{Z}$ nor $\widehat{Z}^{*}$ is contractive, both have attractors as proved in Theorem 5.3 below.

In a metric space, the notation $\mathcal{B}(x, r)$ denotes the open ball of radius $r$ centered at $x$.

\section{Theorem 5.3.}

1. The IFS $\widehat{Z}$ has a point-fibred attractor $\mathbb{I}_{+}$with basin $\mathbb{I} \backslash \mathbb{I}_{-}$and fast basin

$$
\widehat{\mathbb{I}}:=\left\{\iota \in \mathbb{I}: \text { there exists } k \in \mathbb{N} \text { such that } \iota_{j} \in\left\{\begin{array}{l}
I_{-} \text {if } j<k \\
I_{+} \text {if } j \geq k
\end{array}\right\} .\right.
$$

2. The dual IFS $\widehat{Z}^{*}$ has point-fibred attractor $\mathbb{I}_{-}$with basin $\mathbb{I} \backslash \mathbb{I}_{+}$and fast basin

$$
\widehat{\mathbb{I}}^{*}:=\left\{\iota \in \mathbb{I}: \text { there exists } k \in \mathbb{N} \text { such that } \iota_{j} \in\left\{\begin{array}{l}
I_{+} \text {if } j<k \\
I_{-} \text {if } j \geq k
\end{array}\right\} .\right.
$$

Proof. We will show that the IFS $\widehat{Z}$ has point-fibred attractor $\mathbb{I}_{+}$with basin $\mathbb{I} \backslash \mathbb{I}_{-}$and dual repeller $\mathbb{I}_{-}$. The rest of the proof follows immediately, and is omitted. The compact nonempty set $\mathbb{I}_{+}$is contained in $\mathbb{I}$, and $\mathbb{I}_{+}=\widehat{Z}\left(\mathbb{I}_{+}\right):=\cup_{n \in I_{+}} \sigma_{n}\left(\mathbb{I}_{+}\right)$. An open neighborhood of $\mathbb{I}_{+}$is $\mathbb{I} \backslash \mathbb{I}_{-}$. If $\iota \in \mathbb{I} \backslash \mathbb{I}_{-}$, then there is $K=K(\iota) \in \mathbb{N}$ such that $\iota_{K} \in I_{+}$, from which it follows that $\alpha_{1} \in I_{+}$ for all $\alpha \in \widehat{Z}^{K-1}(\{\iota\})$. In turn, this implies

$$
d_{H}\left(\widehat{Z}^{K+j}(\{\iota\}), \mathbb{I}_{+}\right) \leq 2^{-j-1},
$$

for all $j \in \mathbb{N}$. This proves that $\left\{\widehat{Z}^{k}(\{\iota\})\right\}_{k \in \mathbb{N}}$ converges to $\mathbb{I}_{+}$for all $\iota \in \mathbb{I} \backslash \mathbb{I}_{-}$.

Now suppose that $C \in H(\mathbb{I})$ and $C \subset \mathbb{I} \backslash \mathbb{I}_{-}$. Let $\iota \in C$, and let $K=K(\iota)$ be as above. Then for all $\omega \in \mathcal{B}\left(\iota, 2^{-K-1}\right)$ we have $\omega_{K} \in I_{+}$and hence

$$
d_{H}\left(\widehat{Z}^{K(\iota)+j}(\{\omega\}), \mathbb{I}_{+}\right) \leq 2^{-j-1},
$$

which shows that $\left\{\widehat{Z}^{k}\left(\overline{\mathcal{B}\left(\iota, 2^{-K(\iota)-1}\right)}\right)\right\}_{k \in \mathbb{N}}$ converges to $\mathbb{I}_{+}$. Using its compactness, $C$ can be covered by a finite set of such balls, and we conclude that $\left\{\widehat{Z}^{k}(C)\right\}_{k \in \mathbb{N}}$ converges to $\mathbb{I}_{+}$. On the other hand, $\mathbb{I}_{-} \subset \widehat{Z}\left(\mathbb{I}_{-}\right)$so $\mathbb{I}_{-} \subset \widehat{Z}^{k}\left(\mathbb{I}_{-}\right)$for all $k \in \mathbb{N}$, so $\left\{\widehat{Z}^{k}\left(\mathbb{I}_{-}\right)\right\}_{k \in \mathbb{N}}$ does not converge to $\mathbb{I}_{+}$, so we conclude that the basin of the attractor $\mathbb{I}_{+}$(w.r.t. $\widehat{Z}$ ) is $\mathbb{I} \backslash \mathbb{I}_{-}$. Hence, by definition, the dual repeller is $\mathbb{I}_{-}$. 
Theorem 5.4 below is a generalization, along the lines of Kieninger [12, Section 4.2] and Hata [9, Theorem 3.2], to point-fibred attractors, of ideas in Hutchinson's work [10, 2.1.(8), p. 716; 3.1.(3), p. 724]. It asserts, in particular, a semiconjugacy between $\mathcal{F}$ acting on $A$ and the contractive IFS $Z=\left\{\mathbb{I}_{+} ; \sigma_{n}, n \in I_{+}\right\}$of Example 5.1 acting on its attractor, $\mathbb{I}_{+}$.

Theorem 5.4. Let $A$ be a point-fibred attractor of the $\operatorname{IFS} \mathcal{F}=\left\{\mathbb{X} ; f_{n}, n \in I_{+}\right\}$. The map $\pi: \mathbb{I}_{+} \rightarrow A$ from the attractor of $Z$ to the attractor of $\mathcal{F}$ given in equation (2.3) is uniformly continuous, and the diagram

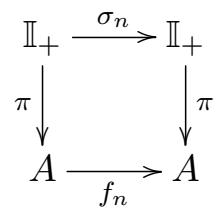

commutes for all $n \in I_{+}$.

Theorem 5.4 describes the behaviour of the functions of the IFS on points of the attractor, not outside the attractor. The results below describe the behaviour of the functions of the IFS on the fast basin of the attractor and, in the case that the IFS is contractive with attractor with nonempty interior, on all of the space $\mathbb{X}$. These new results, Theorem 5.6 and its corollaries, together with Theorem 5.4, provide a more complete symbolic description than the one provided by Theorem 5.4 alone, of the structure and dynamics of point-fibred attractors of IFSs.

Extend the notation of equation (2.2) as follows

$$
\begin{aligned}
& \mathbb{J}_{+}=\left\{\iota \in \mathbb{I}: \exists K \in \mathbb{N}_{0} \text { such that } S^{K}(\iota) \in \mathbb{I}_{+}\right\}, \\
& \mathbb{J}_{-}=\left\{\iota \in \mathbb{I}: \exists K \in \mathbb{N}_{0} \text { such that } S^{K}(\iota) \in \mathbb{I}_{-}\right\},
\end{aligned}
$$

and note that

$$
\mathbb{I}_{+} \subset \widehat{\mathbb{I}} \subset \mathbb{J}_{+} \subset \overline{\mathbb{J}_{+}}=\mathbb{I} \subset \mathbb{I}_{0}, \quad \text { and } \quad \mathbb{I}_{-} \subset \widehat{\mathbb{I}}^{*} \subset \mathbb{J}_{-} \subset \overline{\mathbb{J}_{-}}=\mathbb{I} \subset \mathbb{I}_{0}
$$

In particular, the spaces $\mathbb{J}_{ \pm}$are dense in $\mathbb{I}$. Moreover, $\sigma_{n}\left(\mathbb{J}_{+}\right)=\mathbb{J}_{+}$and $\sigma_{n}\left(\mathbb{J}_{-}\right)=\mathbb{J}_{-}$for all $n \in I$. In addition, $\left.\sigma_{n}\right|_{\mathbb{J}_{+}}: \mathbb{J}_{+} \rightarrow \mathbb{J}_{+}$and $\left.\sigma_{n}\right|_{\mathbb{J}_{-}}: \mathbb{J}_{-} \rightarrow \mathbb{J}_{-}$are homeomorphisms.

Definition 5.5. Let $A$ be a point-fibred attractor of an IFS $\mathcal{F}$. The extended coding map $\pi: \mathbb{J}_{+} \rightarrow \mathbb{X}$ is given by

$$
\pi(\iota)=\lim _{k \rightarrow \infty} f_{\iota \mid k}(b)
$$

for all $\iota \in \mathbb{J}_{+}$, where $b \in B(A)$. The limit in (5.2) exists and is independent of $b$ because, by the definition of $\mathbb{J}_{+}$, there is $K \in \mathbb{N}_{0}$ such that $S^{K}(\iota) \in \mathbb{I}_{+}$, which means that we can write

$$
\pi(\iota)=f_{\iota \mid K}\left(\pi\left(S^{K}(\iota)\right)\right) .
$$

Clearly, the extended coding map agrees with the standard coding map (Definition 2.4) on $\mathbb{I}_{+}$. We will use the same notation for both maps. When the dual repeller $A^{*}$ is a point-fibred attractor of the dual IFS $\mathcal{F}^{*}$, we denote the associated extended coding map by $\pi^{*}: \mathbb{J}_{-} \rightarrow \mathbb{X}$.

Although $\mathbb{I}$ does not serve as a code space - in particular, a result analogous to Theorem 5.4 does not hold - the sets $\mathbb{J}_{ \pm}$, that are dense in $\mathbb{I}$, do. 
Theorem 5.6. Let $A$ be a point-fibred attractor of an IFS $\mathcal{F}$. The extended coding map $\pi: \mathbb{J}_{+} \rightarrow \mathbb{X}$ defined by (5.2) is continuous and agrees with the standard coding map on $\mathbb{I}_{+}$. The left diagram below is commutative for all $n \in I=I_{+} \cup I_{-}$.

If $A^{*}$, the dual repeller of $A$, is a point-fibred attractor of the dual IFS $\mathcal{F}^{*}$, then the right diagram below is commutative for all $n \in I$ :

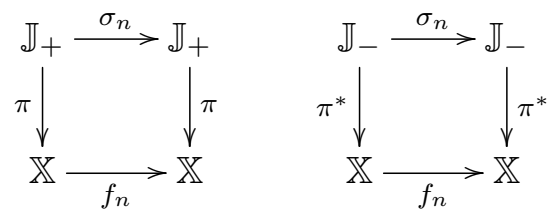

Proof. That the extended coding map $\pi: \mathbb{J}_{+} \rightarrow \mathbb{X}$ agrees with the standard coding map on $\mathbb{I}_{+}$follows immediately from Definitions 2.4 and 5.5. That $\pi$ is continuous follows from the definition in Section 2 of the metric on $\mathbb{I}_{0}$. Concerning the commuting diagram:

$$
\left.\pi\left(\sigma_{n}(\iota)\right)=\lim _{k \rightarrow \infty} f_{n} \circ f_{\iota \mid k}(b)\right)=f_{n}\left(\lim _{k \rightarrow \infty} f_{\iota \mid k}(b)\right)=f_{n}(\pi(\iota)) .
$$

The proof is similar for the dual.

Example 5.7. If $\mathcal{F}$ is as in Example 3.6, then the ranges of both $\pi$ and $\pi^{*}$ are contained in $\Lambda$.

Corollary 5.8. If $\mathcal{F}$ is an IFS with point-fibred attractor $A$ and fast basin $\widehat{B}$, then $\pi: \widehat{\mathbb{I}} \rightarrow \widehat{B}$ is surjective and the left diagram below is commutative for all $n \in I_{-}$.

Let $\widehat{B}^{*}$ denote the fast basin of the dual IFS $\mathcal{F}^{*}$. If $A^{*}=\mathbb{X} \backslash B(A)$ is a point-fibred attractor of the dual IFS $\mathcal{F}^{*}$, then $\pi^{*}: \widehat{\mathbb{I}}^{*} \rightarrow \widehat{B}^{*}$ is surjective and the right diagram below is commutative for all $n \in I_{+}$.
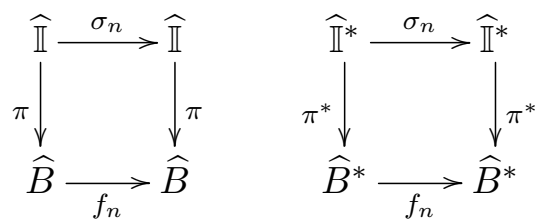

Proof. This is a corollary of Theorem 5.6. We simply note that $\sigma_{-n}(\widehat{\mathbb{I}}) \subset \widehat{\mathbb{I}}$ and $\sigma_{n}\left(\widehat{\mathbb{I}}^{*}\right) \subset \widehat{\mathbb{I}}^{*}$ for all $n \in I_{+}$.

The following corollary provides a model for attractor-repeller pairs, for example the loxodromic Möbius case discussed in [17].

Corollary 5.9. Let $\mathcal{F}$ be an IFS with point-fibred attractor $A$ on a complete metric space $X$ such that $A^{\circ} \neq \varnothing$. If $\mathcal{F}$ is contractive, then $\pi(\widehat{\mathbb{I}})=\mathbb{X}$ and the left diagram below commutes for all $n \in I_{-}$. If $\mathbb{X}$ is compact, let the dual repeller $A^{*}$ be a point-fibred attractor of $\mathcal{F}^{*}$ with $\left(A^{*}\right)^{\circ} \neq \varnothing$. Then $\pi(\widehat{\mathbb{I}})=\pi^{*}\left(\widehat{\mathbb{I}}^{*}\right)=\mathbb{X}$; the left diagram commutes for all $n \in I_{-}$, and the right diagram commutes for all $n \in I_{+}$.
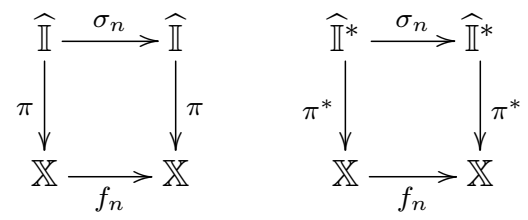

Proof. The result follows from Corollary 5.8 once it is shown that the fast basin of $A$ is $\mathbb{X}$ and the fast basin of $A^{*}$ is also $\mathbb{X}$. In the case of a contractive IFS with $A^{\circ} \neq \varnothing$, the fast basin and the basin coincide and both equal $\mathbb{X}$; see Section 2 . The dual repeller in this case is empty. 
In the $\mathbb{X}$ compact case, we will show that the fast basin of $A^{*}$ is $\mathbb{X}$; a similar argument shows that the fast basin of $A$ is $\mathbb{X}$. It is known [7, Theorem 5.2] that the basin $B\left(A^{*}\right)=\mathbb{X} \backslash A$. So by Proposition 2.7 and Theorems 4.5 and 4.4, if $x \notin A$, then $x$ is in the fast basin of $A^{*}$. Lastly, if $x \in A$, let $y \neq x$ be any other point of $A$. If $\iota$ is any address of $y$, then $\pi(\iota)=\lim _{k \rightarrow \infty} f_{\iota \mid k}(A)$. Therefore there is a $k$ such that $x \notin f_{\iota \mid k}(A)$, which implies that

$$
z:=f_{\iota_{k}}^{-1} \circ f_{\iota_{k-1}}^{-1} \circ \cdots \circ f_{\iota_{1}}^{-1}(y) \notin A .
$$

Since $z$ is in the basin of $A^{*}$ and $\left(A^{*}\right)^{\circ} \neq \varnothing$, there is $\omega_{1} \omega_{2} \cdots \omega_{j}$ for some $j$ such that $f_{\omega_{1}}^{-1} \circ f_{\omega_{2}}^{-1} \circ$ $\cdots \circ f_{\omega_{j}}^{-1}(z) \in A$. Hence $x$ lies in the fast basin of $A^{*}$.

Theorem 5.6 and its corollaries have many consequences including the following: (i) the feasibility of continuous assignment of addresses to points in $\widehat{B}(A)$; (ii) symbolic dynamics on $\widehat{\mathbb{I}}$ are semiconjugate to corresponding dynamics on $\widehat{B}(A)$; (iii) if $A$ has nonempty interior as a subset of $\mathbb{X}$, then we obtain addresses and dynamics on the basin $B(A)$; (iv) description of the relationship between attractors and dual repellers; (v) extension of fractal homeomorphisms, generalizing results in [1] and [8]; (vi) address structures for continuations, branched fractal manifolds and tilings, thereby clarifying and extending ideas in [8].

\section{Fractal manifold}

Throughout this section, $A$ is a point-fibred attractor of an IFS $\mathcal{F}$ on a complete metric space $\mathbb{X}$. The branched fractal manifold generated by $(\mathcal{F}, A)$ is constructed in this section. Properties of the branched fractal manifold are the subject of Theorem 6.4.

Definition 6.1. For $\iota \in \widehat{\mathbb{I}}$, let

$$
k_{\iota}=\min \left\{k: \pi\left(S^{k}(\iota)\right) \in A\right\} \in \mathbb{N}_{0} .
$$

Define

$$
[\iota]=\iota \mid k_{\iota} \quad \text { and } \quad\langle\iota\rangle=S^{k_{\iota}}(\iota),
$$

to be the integer part and the fractional part of the address $\iota=\iota_{1} \iota_{2} \cdots \iota_{k_{\iota}} \cdots$ (w.r.t. $\mathcal{F}$ and $A$ ) respectively.

Example 6.2 (integer and fractional parts). Consider the case $\mathcal{F}=\left\{\mathbb{R} ; f_{1}, f_{2}\right\}$, where $f_{1}(x)=$ $\frac{1}{2} x$ and $f_{2}(x)=\frac{1}{2} x+\frac{1}{2}$. The unique attractor is $A=[0,1]$. We will determine the integer and fractional parts of the two elements of $\widehat{\mathbb{I}}$, namely $-1-1-1 \overline{2}$ and $-1-1-21 \overline{2}$. A "bar" over a sequence means infinite repetition; in particular $\overline{2}=222 \cdots$. Noting that $\pi(\overline{2})=1 \in \mathbb{R}$ and $\pi(-1 \overline{2})=2 \in \mathbb{R}$ we have

$$
[-1-1-1 \overline{2}]=-1-1-1, \quad\langle-1-1-1 \overline{2}\rangle=\overline{2} .
$$

Noting that $\pi(-1-1-21 \overline{2})=0 \in \mathbb{R}$, we have

$$
[-1-1-21 \overline{2}]=\varnothing, \quad\langle-1-1-21 \overline{2}\rangle=-1-1-21 \overline{2} .
$$

Define an equivalence relation $\sim$ on $\widehat{\mathbb{I}}$ by $\iota \sim \omega$ if and only if

1) $[\iota]=[\omega]$, and

2) $\pi(\iota)=\pi(\omega)$, 
and let

$$
\mathbb{L}=\widehat{\mathbb{I}} / \sim
$$

For $\iota \in \widehat{\mathbb{I}}$, let $\widetilde{\iota}$ denote the equivalence class to which $\iota$ belongs. Maps $\widetilde{\pi}: \widehat{\mathbb{I}} \rightarrow \mathbb{L}$ and $\widehat{\pi}: \mathbb{L} \rightarrow \mathbb{X}$ are well defined by $\widetilde{\pi}(\iota)=\widetilde{\iota}$ and $\widehat{\pi}(\widetilde{\iota})=\pi(\iota)$, respectively. The maps $\widehat{\pi}$ and $\widetilde{\pi}$ will be called projection maps.

For $\alpha:=\widetilde{\iota} \in \mathbb{L}$, let

$$
k_{\alpha}:=k_{\iota}, \quad[\alpha]:=[\iota], \quad\langle\alpha\rangle:=\widetilde{\langle\iota\rangle} .
$$

These entities do not depend on the representative $\iota$ of the equivalence class $\alpha$. It is useful to think of $\mathbb{L}$ in terms of leaves, panicles, and sheets.

Definition 6.3. If $\theta$ is a finite word in the alphabet $I_{-}$, then

$$
l(\theta)=\{\alpha \in \mathbb{L}:[\alpha]=\theta\}
$$

will be called a leaf of $\mathbb{L}$, and

$$
p(\theta)=l(\varnothing) \cup l\left(\theta_{1}\right) \cup l\left(\theta_{1} \theta_{2}\right) \cup \cdots \cup l(\theta)
$$

will be called a panicle of $\mathbb{L}$. Because of the special importance of the case $\theta=\varnothing$, define

$$
\mathbb{K}:=l(\varnothing)=p(\varnothing)
$$

If $\theta \in \mathbb{I}_{-}$, we will refer to the "infinite panicle"

$$
\mathbb{L}_{\theta}:=\bigcup_{k=0}^{\infty} l(\theta \mid k)
$$

as a sheet of $\mathbb{L}$.

Define a metric on $\mathbb{L}$ as follows. That it is a metric is part of Theorem 6.4 below. For $\alpha, \beta \in \mathbb{L}$ let

$$
K:=\max \{k:[\alpha]|k=[\beta]| k\}, \quad[\alpha, \beta]:=[\alpha]|K=[\beta]| K, \quad p(\alpha, \beta):=p([\alpha, \beta]),
$$

and

$$
d_{\mathbb{L}}(\alpha, \beta)=\min \left\{d_{\mathbb{X}}(\widehat{\pi}(\alpha), \widehat{\pi}(\iota))+d_{\mathbb{X}}(\widehat{\pi}(\iota), \widehat{\pi}(\beta)): \iota \in p(\alpha, \beta)\right\} .
$$

Because $p(\alpha, \beta)=f_{[\alpha, \beta]}(A)$, where the notation of equation 2.1 is used, it is equivalent to define

$$
d_{\mathbb{L}}(\alpha, \beta)=\min \left\{d_{\mathbb{X}}(\widehat{\pi}(\alpha), x)+d_{\mathbb{X}}(x, \widehat{\pi}(\beta)): x \in f_{[\alpha, \beta]}(A)\right\} .
$$

The metric space $\left(\mathbb{L}, d_{\mathbb{L}}\right)$ will be called the branched fractal manifold or just the $f$-manifold generated by $(\mathcal{F}, A)$. A description of the f-manifold is the intent of the following theorem. Shift maps $\widetilde{\sigma}_{n}: \mathbb{L} \rightarrow \mathbb{L}$ are well-defined by

$$
\widetilde{\sigma}_{n}(\widetilde{\iota})=\widetilde{\sigma_{n}(\iota)} .
$$

These maps occur in statement (9). 
Theorem 6.4. Let $A$ be a point-fibred attractor of an IFS $\mathcal{F}$ with fast basin $\widehat{B} \subset \mathbb{X}$. Let $\left(\mathbb{L}, d_{\mathbb{L}}\right)$ be the $f$-manifold generated by $(\mathcal{F}, A)$. Let $\widehat{\pi}: \mathbb{L} \rightarrow \mathbb{X}$ and $\widetilde{\pi}: \widehat{\mathbb{I}} \rightarrow \mathbb{L}$ be the associated projection maps. The following statements hold.

1. The minimum in equation (6.1) is achieved, and $d_{\mathbb{L}}$ is a metric on $\mathbb{L}$.

2. The set of all leaves form a partition of $\mathbb{L}$, and each leaf is nonempty.

3. The image of $\mathbb{L}$ under $\widehat{\pi}$ is the fast basin, i.e., $\widehat{\pi}(\mathbb{L})=\widehat{B}$.

4. For any $\theta \in \mathbb{I}_{-}$, the projection $\widehat{\pi}$ maps the sheet $\mathbb{L}_{\theta}$ isometrically onto the continuation $B_{-\theta} \subseteq \widehat{B} \subseteq \mathbb{X}$ :

$$
d_{\mathbb{X}}(\widehat{\pi}(\alpha), \widehat{\pi}(\beta))=d_{\mathbb{L}}(\alpha, \beta) .
$$

for all $\alpha, \beta \in \mathbb{L}_{\theta}$.

5. Each panicle is homeomorphic to $A$.

6. The projection maps $\tilde{\pi}: \widehat{\mathbb{I}} \rightarrow \mathbb{L}$ and $\widehat{\pi}: \mathbb{L} \rightarrow \widehat{B}$, are continuous; in particular

$$
d_{\mathbb{X}}(\widehat{\pi}(\alpha), \widehat{\pi}(\beta)) \leq d_{\mathbb{L}}(\alpha, \beta) .
$$

for all $\alpha, \beta \in \mathbb{L}$.

7. If $A$ is pathwise connected, then $\mathbb{L}$ is pathwise connected.

8. If the metric spaces $\left(\mathbb{X}, d_{\mathbb{X}}\right)$ and $\left(\mathbb{X}, d_{\mathbb{X}}^{\prime}\right)$ have the same topology, then $\left(\mathbb{L}, d_{\mathbb{L}}\right)$ and $\left(\mathbb{L}, d_{\mathbb{L}}^{\prime}\right)$ have the same topology, where the metric $d_{\mathbb{L}}^{\prime}$ is defined by equation (6.1) with $d_{\mathbb{X}}$ replaced by $d_{\mathbb{X}}^{\prime}$.

9. With $\sigma_{n}: \widehat{\mathbb{I}} \rightarrow \widehat{\mathbb{I}}$ as defined in Section 5 and $\widetilde{\sigma}_{n}: \mathbb{L} \rightarrow \mathbb{L}$ as defined above, both $\sigma_{n}$ and $\widetilde{\sigma}_{n}$ are continuous and injective, for all $n \in I_{-}$, and we have the commutative diagram,

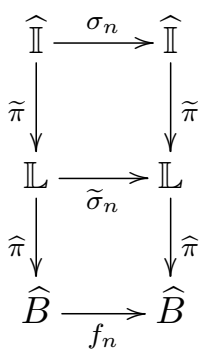

Proof. The proofs of (2), (3), (9) are straightforward.

(1) The set $f_{[\alpha, \beta]}(A)$ in (6.2) is compact because $A$ is compact and $f_{[\alpha, \beta]}$ is continuous. For fixed $\alpha, \beta$, the function $d_{\mathbb{X}}(\widehat{\pi}(\alpha), x)+d_{\mathbb{X}}(x, \widehat{\pi}(\beta))$ is continuous in $x \in f_{[\alpha, \beta]}(A)$. Hence the minimum is achieved at some $x^{*} \in f_{[\alpha, \beta]}(A)$.

Concerning the metric, we establish only the triangle inequality:

$$
d_{\mathbb{L}}(\alpha, \gamma)+d_{\mathbb{L}}(\gamma, \beta)=d_{\mathbb{X}}\left(\widehat{\pi}(\alpha), x_{1}\right)+d_{\mathbb{X}}\left(x_{1}, \widehat{\pi}(\gamma)\right)+d_{\mathbb{X}}\left(\widehat{\pi}(\gamma), x_{2}\right)+d_{\mathbb{X}}\left(x_{2}, \widehat{\pi}(\beta)\right),
$$

where $x_{1} \in f_{[\alpha, \gamma]}(A)$ minimizes $d_{\mathbb{X}}\left(\widehat{\pi}(\alpha), x_{1}\right)+d_{\mathbb{X}}\left(x_{1}, \widehat{\pi}(\gamma)\right)$ and $x_{2} \in f_{[\beta, \gamma]}(A)$ minimizes $d_{\mathbb{X}}\left(\widehat{\pi}(\gamma), x_{2}\right)+d_{\mathbb{X}}\left(x_{2}, \widehat{\pi}(\beta)\right)$. We have either $f_{[\alpha, \gamma]}(A) \subset f_{[\alpha, \beta]}(A)$ or $f_{[\beta, \gamma]}(A) \subset f_{[\alpha, \beta]}(A)$. Without loss of generality assume that $f_{[\beta, \gamma]}(A) \subset f_{[\alpha, \beta]}(A)$. Then

$$
\begin{aligned}
d_{\mathbb{L}}(\alpha, \gamma)+d_{\mathbb{L}}(\gamma, \beta) & =d_{\mathbb{X}}\left(\widehat{\pi}(\alpha), x_{1}\right)+d_{\mathbb{X}}\left(x_{1}, \widehat{\pi}(\gamma)\right)+d_{\mathbb{X}}\left(\widehat{\pi}(\gamma), x_{2}\right)+d_{\mathbb{X}}\left(x_{2}, \widehat{\pi}(\beta)\right) \\
& \geq d_{\mathbb{X}}\left(\widehat{\pi}(\alpha), x_{1}\right)+d_{\mathbb{X}}\left(x_{1}, x_{2}\right)+d_{\mathbb{X}}\left(x_{2}, \widehat{\pi}(\beta)\right) \\
& \geq d_{\mathbb{X}}\left(\widehat{\pi}(\alpha), x_{2}\right)+d_{\mathbb{X}}\left(x_{2}, \widehat{\pi}(\beta)\right)\left(\text { where } x_{2} \in f_{[\beta, \gamma]}(A)\right) \\
& \geq \min \left\{d_{\mathbb{X}}(\widehat{\pi}(\alpha), x)+d_{\mathbb{X}}(x, \widehat{\pi}(\beta)): x \in f_{[\alpha, \beta]}(A)\right\} \\
& =d_{\mathbb{L}}(\alpha, \beta) .
\end{aligned}
$$


(4) Assume that $\alpha, \beta \in \mathbb{L}$ lie in the same sheet, and therefore lie in a common panicle, say $p([\beta])$. Then $[\alpha, \beta]=[\alpha]$ and $f_{[\alpha, \beta]}(A)=f_{[\alpha]}(A)$, which implies that $\widehat{\pi}(\alpha) \in f_{[\alpha]}(A)$. By the triangle inequality we have $d_{\mathbb{L}}(\alpha, \beta)=\min \left\{d_{\mathbb{X}}(\widehat{\pi}(\alpha), x)+d_{\mathbb{X}}(x, \widehat{\pi}(\beta)): x \in f_{[\alpha, \beta]}(A)\right\}=$ $d_{\mathbb{X}}(\widehat{\pi}(\alpha), \widehat{\pi}(\beta))$. That the image of $\mathbb{L}_{\theta}$ under $\widehat{\pi}$ is $B_{-\theta}$ follows from the definitions of $\widehat{\pi}$ and of the continuation $B_{-\theta}$.

(5) It follows from the definitions that $\widehat{\pi}$ maps the panicle $p(\theta)$ onto $f_{\theta}(A)$. The result now follows from that facts that $f_{\theta}$ is a homeomorphism and that, by part (4), the mapping $\widehat{\pi}$ restricted to $p(\theta)$ is an isometry.

(6) The continuity of $\widehat{\pi}: \mathbb{L} \rightarrow \mathbb{X}$ follows from the inequality $d_{\mathbb{X}}(\widehat{\pi}(\alpha), \widehat{\pi}(\beta)) \leq d_{\mathbb{L}}(\alpha, \beta)$, which follows from the triangle inequality.

Concerning the continuity of $\tilde{\pi}: \widehat{\mathbb{I}} \rightarrow \mathbb{L}$, let $\iota \in \widehat{\mathbb{I}}$ be given. If $\omega \in \widehat{\mathbb{I}}$ and $\omega|K=\iota| K$ for $K$ sufficiently large, that is, if $d_{\mathbb{I}}(\iota, \omega)$ is sufficiently small, then $\widetilde{\omega}$ and $\widetilde{\iota}$ lie the same leaf. By part $(4) d_{\mathbb{L}}(\widetilde{\pi}(\iota), \widetilde{\pi}(\omega))=d_{\mathbb{X}}(\widehat{\pi}(\widetilde{\iota}), \widehat{\pi}(\widetilde{\omega}))=d_{\mathbb{X}}(\widehat{\pi}(\iota), \widehat{\pi}(\omega))$. The continuity of $\widetilde{\pi}: \widehat{\mathbb{I}} \rightarrow \mathbb{L}$ then follows from the continuity of $\widehat{\pi}: \widehat{\mathbb{I}} \rightarrow \mathbb{X}$ by Theorem 5.6.

(7) Assume that $A$ is pathwise connected. Given any two points $\alpha, \beta \in \mathbb{L}$, the leaf $\mathbb{K}$ is contained in the intersection of the sheets containing $\alpha$ and $\beta$. It is therefore sufficient to prove that any sheet is pathwise connected; so assume that $\alpha$ and $\beta$ lie in the same sheet, which implies that they lie in the same panicle $p(\theta)$ for some finite word $\theta$. But by (5), this panicle is homeomorphic to $A$.

(8) Two metrics $d_{\mathbb{X}}$ and $d_{\mathbb{X}}^{\prime}$ on $\mathbb{X}$ generate the same topology if, given any ball $\mathcal{B}_{\left(\mathbb{X}, d_{\mathbb{X}}\right)}(x, r)$, $r>0$, there is a positive radius $r^{\prime}$ such that $\mathcal{B}_{\left(\mathbb{X}, d_{\mathbb{X}}^{\prime}\right)}\left(x, r^{\prime}\right) \subset \mathcal{B}_{\left(\mathbb{X}, d_{\mathbb{X}}\right)}(x, r)$ and, given any $\mathcal{B}_{\left(\mathbb{X}, d_{\mathbb{X}}^{\prime}\right)}\left(x, r^{\prime}\right)$, there is a positive radius $r$ such that $\mathcal{B}_{\left(\mathbb{X}, d_{\mathbb{X}}\right)}(x, r) \subset \mathcal{B}_{\left(\mathbb{X}, d_{\mathbb{X}}^{\prime}\right)}\left(x, r^{\prime}\right)$. In the present situation, let the metrics on $\mathbb{L}$ defined using $d_{\mathbb{X}}$ and $d_{\mathbb{X}}^{\prime}$ be denoted by $d_{\mathbb{L}}$ and $d_{\mathbb{L}}^{\prime}$. Let $r>0$ be given and $\alpha \in \mathbb{L}$ be given. We will show that there is $r^{\prime}>0$ so that $\mathcal{B}_{\left(\mathbb{L}, d_{\mathbb{L}}^{\prime}\right)}\left(\alpha, r^{\prime}\right) \subset \mathcal{B}_{\left(\mathbb{L}, d_{\mathbb{L}}\right)}(\alpha, r)$. The rest of the argument is then obtained by switching the roles of the key players.

If $\alpha$ and $\beta$ are contained in the same sheet, then by (4), we have $d_{\mathbb{L}}(\alpha, \beta)=d_{\mathbb{X}}(\widehat{\pi}(\alpha), \widehat{\pi}(\beta))$, so that

$$
\mathcal{B}_{\left(\mathbb{L}, d_{\mathbb{L}}\right)}(\alpha, r)=\left\{\beta \in \mathbb{L}: \widehat{\pi}(\beta) \in \mathcal{B}_{\left(\mathbb{X}, d_{\mathbb{X}}\right)}(\widehat{\pi}(\alpha), r)\right\} .
$$

Now choose $r^{\prime}>0$ so small that

$$
\mathcal{B}_{\left(\mathbb{X}, d_{\mathbb{X}}^{\prime}\right)}\left(\widehat{\pi}(\alpha), r^{\prime}\right) \subset \mathcal{B}_{\left(\mathbb{X}, d_{\mathbb{X}}\right)}(\widehat{\pi}(\alpha), r) .
$$

Then, if $r^{\prime}$ is sufficiently small,

$$
\begin{aligned}
\mathcal{B}_{\left(\mathbb{L}, d_{\mathbb{L}}\right)}\left(\alpha, r^{\prime}\right) & =\left\{\beta \in \mathbb{L}: \widehat{\pi}(\beta) \in \mathcal{B}_{\left(\mathbb{X}, d_{\mathbb{X}}\right)}\left(\widehat{\pi}(\alpha), r^{\prime}\right)\right\} \\
& \subset\left\{\beta \in \mathbb{L}: \widehat{\pi}(\beta) \in \mathcal{B}_{\left(\mathbb{X}, d_{\mathbb{X}}\right)}(\widehat{\pi}(\alpha), r)\right\}=\mathcal{B}_{\left(\mathbb{L}, d_{\mathbb{L}}\right)}(\alpha, r) .
\end{aligned}
$$

It now suffices to show that if $\alpha$ is fixed and $\beta$ is sufficiently close to $\alpha$, then $\alpha$ and $\beta$ lie on the same sheet. Assume that $\left\{\beta_{n}\right\}$ is a sequence of points in $\mathbb{L}$ that converges to $\alpha$, but $\beta_{n}$ is not in the same sheet as $\alpha$ for every $n$. By the definition of the distance on the f-manifold, this implies that there is a sequence $\left\{\gamma_{n}\right\}$ such that $\gamma_{n} \in p\left(\alpha, \beta_{n}\right)$ and $\widehat{\pi}\left(\gamma_{n}\right)$ converges to $\widehat{\pi}(\alpha)$. Because $\alpha$ is fixed, the set $\left\{p\left(\alpha, \beta_{n}\right)\right\}$ has at most finitely many distinct elements. So there is an infinite subsequence $\left\{\gamma_{n}^{\prime}\right\}$ of $\left\{\gamma_{n}\right\}$ and the corresponding subsequence $\left\{\beta_{n}^{\prime}\right\}$ of $\left\{\beta_{n}\right\}$ such that

$$
\gamma_{n}^{\prime} \in p\left(\alpha, \beta_{n}^{\prime}\right)=p\left(\alpha, \beta_{0}\right)
$$

for some fixed $\beta_{0}$. Since $\alpha, \gamma_{n}^{\prime} \in p(\alpha)$ for all $n$ and $p(\alpha)$ is compact by statement (5), it follows from the fact that $\widehat{\pi}\left(\gamma_{n}^{\prime}\right)$ converges to $\widehat{\pi}(\alpha)$ and statement (4) that $\left\{\gamma_{n}\right\}$ converges to $\alpha$. But from the facts that $p\left(\alpha, \beta_{0}\right)$ is compact and $\gamma_{n}^{\prime} \in p\left(\alpha, \beta_{0}\right)$, it follows that $\alpha \in p\left(\alpha, \beta_{0}\right)$. Therefore, $\alpha$ and $\beta_{n}^{\prime}$ are in the same panicle, and hence the same sheet, for all $n$, a contradiction. 


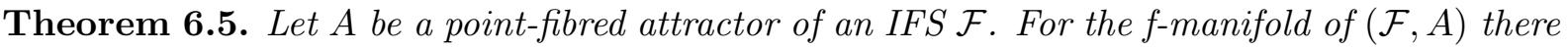
are at most $N+1$ topologically distinct leaves. Specifically, the leaf $l(\varnothing)=\mathbb{K}$ is homeomorphic to $A$, and the leaf $l(\theta)$ for $\theta \neq \varnothing$ is homeomorphic to $A \backslash f_{i_{\theta}}(A)$ where $-i_{\theta}$ is the last component of $\theta$.

Proof. To simplify notation, when the intent is clear, we make no distiction between $\iota \in \widehat{\mathbb{I}}$ and its equivalence class in $\mathbb{L}$. For $\alpha \in \mathbb{L}$, note that $\pi(\langle\alpha\rangle) \in A$. For a leaf $l(\theta)$, let $\langle l(\theta)\rangle=\{\langle\alpha\rangle: \alpha \in$ $l(\theta)\}$. By definition, $\iota \in\langle l(\theta)\rangle$ if and only if $\pi(\iota) \in A$ and $f_{S^{i}(\iota)}(\pi(\iota)) \notin A$ for $i=0,1,2, \ldots, k_{\iota}-1$; the last condition here is equivalent to $f_{i_{\theta}}(\pi(\iota)) \notin A$ where $-i_{\theta}$ is the last entry in $\theta$. Therefore $\pi(\langle l(\theta)\rangle)=A \backslash f_{i_{\theta}}(A)$. This implies that $\widehat{\pi}(l(\theta))=f_{\theta}(\pi(\langle l(\theta)\rangle))=f_{\theta}\left(A \backslash f_{i_{\theta}}(A)\right)$. Since $\widehat{\pi}$ restricted to $l(\theta)$ is a homeomorphism by part (4) of Theorem 6.4, and $f_{\theta}$ is a homeomorphism, we have $l(\theta)$ is homeomorphic to $A \backslash f_{i_{\theta}}(A)$.

Corollary 6.6. Let $A$ be a point-fibred attractor of an IFS $\mathcal{F}$. The closures of any two distinct leaves of the f-manifold are non-overlapping.

Proof. This follows from part (5) of Theorem 6.4, Theorem 6.5, and the fact that the boundary of a compact set has empty interior.

Example 6.7. The attractor of the IFS $\left\{\mathbb{R} ; f_{1}, f_{2}\right\}$ where $f_{1}(x)=\frac{1}{3} x$ and $f_{2}(x)=\frac{1}{3} x+\frac{2}{3}$, is the Cantor set $\mathcal{C}$. The f-manifold $\mathbb{L}$ is the disjoint union of its leaves, each of which is homeomorphic to $\mathcal{C}$. The image of each leaf under the projection map $\widehat{\pi}$ is related by a similitude to $\mathcal{C}$, with scaling factor equal to $3^{k}$ for some $k \in \mathbb{N}_{0}$.

Example 6.8. The attractor of the IFS $\left\{\mathbb{R} ; f_{1}, f_{2}\right\}$ where $f_{1}(x)=\frac{1}{2} x$ and $f_{2}(x)=\frac{1}{2} x+\frac{1}{2}$, is the unit interval $A=[0,1]$. The f-manifold is a connected, branched 1-manifold. The set $\mathbb{K}=l(\varnothing)$ is homeomorphic to $A=[0,1]$. According to Theorem 6.5, all other leaves are homeomorphic to half open intervals. Each panicle, a union of leaves, is homeomorphic to a closed interval. Routine calculation shows the following. The length of the projection of each leaf, under $\widehat{\pi}$, is a power of 2 . Each point $\alpha \in \mathbb{L}$ is contained in a neighborhood that is homeomorphic to the open interval $(0,1)$ or, if it is a branch point, to the union of a countably infinite number of copies of $[0,1)$ where only $\alpha$ is common to the copies. The branch points are isolated. More exactly, the branch points are the points of $\mathbb{L}$ whose projection under $\widehat{\pi}$ are of the form $2^{n}, n \geq 0$, or $-2^{n}+1, n \geq 0$. Each sheet of $\mathbb{L}$ is mapped isometrically by $\widehat{\pi}$ to either $\mathbb{R}$ or the interval $[0, \infty)$ or the interval $(-\infty, 1]$. For example (recall that the "bar" means infinite repetition) the sheet $\mathbb{L} \frac{}{-2-1}$ is homeomorphic to $\mathbb{R}$, and $\mathbb{L} \frac{-2}{-2}$ is homeomorphic to $(-\infty, 1]$. See Fig. 4.

Example 6.9. While it is easy to visualize the branched fractal manifold in the above example, it is harder to picture it in the following example, illustrated in Fig. 5. Here the IFS $\mathcal{F}=$ $\left\{\mathbb{R}^{2} ; f_{n}, n=1,2,3,4\right\}$ is affine, with the following property. There is a triangle $A B C$ with points $c \in A B, a \in B C, b \in C A$, where $X Y$ is the line segment joining the points $X$ and $Y$, and the triangles $a b c, A B C, A b c, a B c, a b C$ are non-degenerate. Moreover, $f_{1}(A B C)=A b c$, $f_{2}(A B C)=a B c, f_{3}(A B C)=a b C$, and $f_{4}(A B C)=a b c$. IFSs of this kind are discussed in [1]. This IFS possesses a unique attractor, the filled triangle with vertices $A B C$. This attractor is represented, in each of the four panels of Fig. 5, by a very small multicoloured triangle located near the center of each of the four pictures; directly below it is a small yellow triangle. If $\theta \in \mathbb{I}_{+}$is reversible, then $\widehat{B}_{\theta}=\mathbb{R}^{2}$. The branched fractal manifold consists of non-denumerably many copies of $\mathbb{R}^{2}$ glued together appropriately. Each region of glue is triangular. The top left panel illustrates $B_{i j k 1}$ for a fixed choice of $i j k$. It comprises 256 copies of $A$, namely the sets $f_{i}^{-1} \circ f_{j}^{-1} \circ f_{k}^{-1} \circ f_{1}^{-1} \circ f_{m} \circ f_{n} \circ f_{o} \circ f_{p}(A)$ for all $m, n, o, p \in\{1,2,3,4\}$, each copy in a colour that is supposed to be different from its neighbours. The top right panel illustrates $B_{i j k 1} \cup B_{i j k 2}$, where each of the subtriangles $f_{i}^{-1} \circ f_{j}^{-1} \circ f_{k}^{-1} \circ f_{2}^{-1} \circ f_{m} \circ f_{n} \circ f_{o} \circ f_{p}(A)$ for all $m, n, o, p \in\{1,2,3,4\}$ 


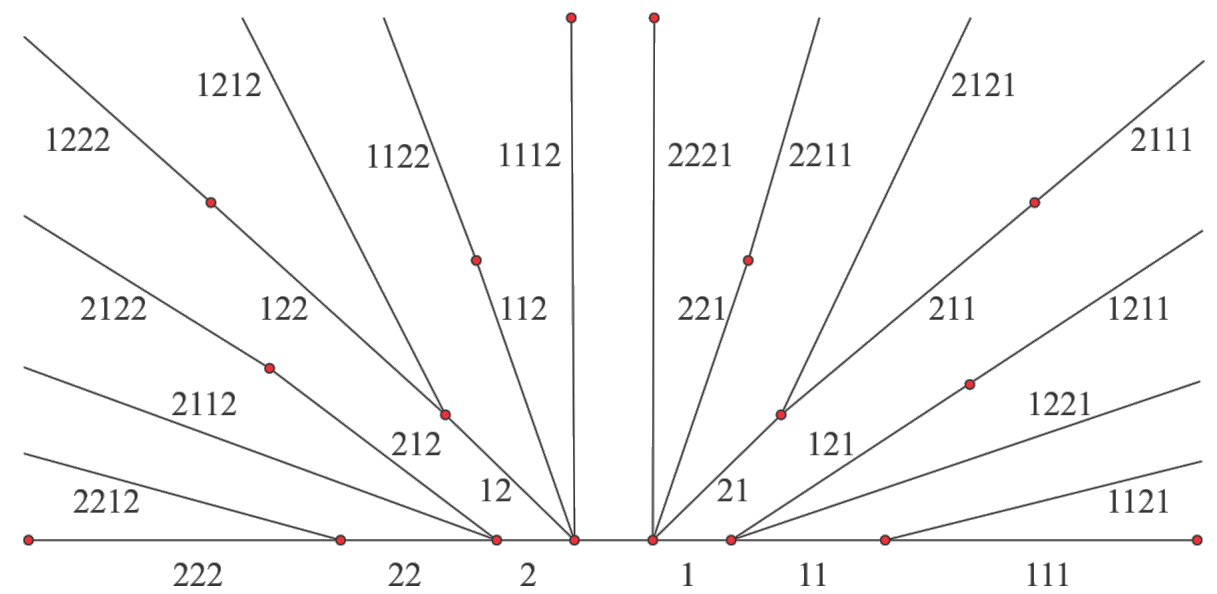

Figure 4. A portion of the f-manifold of Example 6.8. Each leaf $l(\theta)$ is labeled by $-\theta$. Note that there are additional leaves, with longer labels, incident with some of the branch points in the diagram, that are not included in the diagram.

is coloured so that it is different from its neighbours. The two images may be thought to be glued together on $B_{i j k}$. Similarly, the bottom left panel illustrates $B_{i j k 1} \cup B_{i j k 2} \cup B_{i j k 3}$ and the bottom right panel illustrates $B_{i j k 1} \cup B_{i j k 2} \cup B_{i j k 3} \cup B_{i j k 4}$.

\section{Related work}

Fast basins, the extended code space, and branched fractal manifolds were first introduced in the earliest version of this paper [3], which also concerns the notion of fractal continuation, first introduced in [6].

The notion of fractal continuation is discussed in [5, 6], in the context of fractal interpolation, and in [8] in the context of tiling theory. The scope of the present work is much wider, most particularly with regard to the definition and topology of fast basins and branched fractal manifolds. In [2], a work in progress, conditions under which certain topological and geometrical properties that the fast basin inherits from the attractor of the IFS are established. These properties include the Hausdorff and topological dimension, local connectivity and $\sigma$-porosity.

Notions related to but distinct from fast basins and branched fractal manifolds occur in the literature, mainly in the context of IFSs of similitudes with the open set condition (OSC). In particular, some fast basins are fractafolds as defined by Stritchartz [16, Section 5.4], and some are examples of fractafold bundles as defined by Ionescu and Kumjian [11]. We discuss these related notions in more detail in the following paragraphs.

In [14] Strichartz defines and establishes some properties of fractal blowups (called fractal continuations here) for an IFS consisting of similitudes that obeys the OSC. Fractal blowups for the classical Cantor set, the Sierpinski triangle, and the Koch curve are investigated, and it is noted that various different IFSs, with the Koch curve as the attractor, yield different blowups. It is pointed out that in general a given IFS may yield a nondenumerable set of nonhomeomorphic blowups, where the relative topology is assumed. Strichartz is focused on post critically finite (PCF) fractals because energy forms and Laplacians can be defined on their blowups. In [16, Section 5.4] Strichartz defines a fractafold to be a countable union of PCF fractals, glued together on their boundaries in such a way that every point in the fractafold has a neighborhood that is conjugate to a neighborhood of a point on the attractor; this notion subsumes fractal blowups. In his context, Strichartz considers a fractafold to be a generalization of the notion of a manifold, with the role of Euclidean space (or half-space) being played by the 


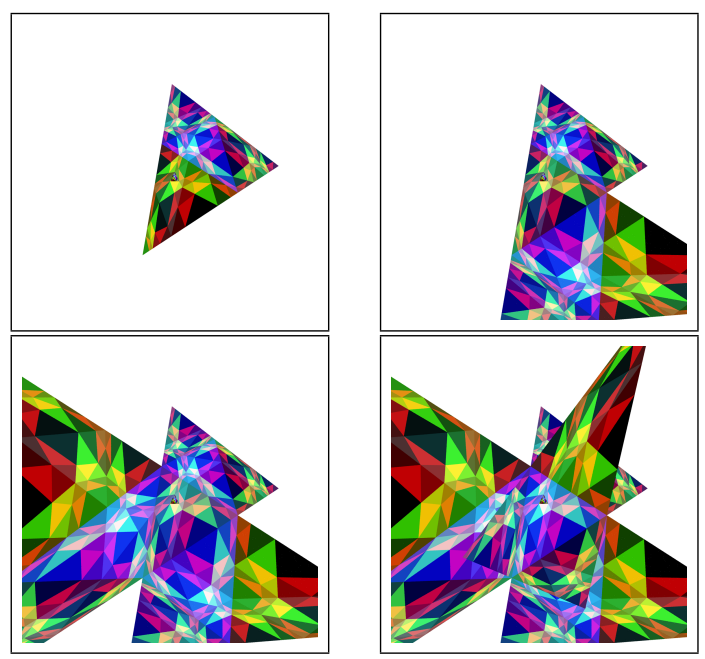

Figure 5. Fractal manifold under construction. See Example 6.9.

attractor. In various works, see for example [15], he has shown how energy forms and Laplacians on PCF fractals can be extended to fractafolds.

Some branched fractal manifolds are related to fractafold bundles, defined by Ionescu and Kumjian [11]. They consider IFSs of the form of $\mathcal{F}$ with $0<r_{i} \leq R_{i}<1$ and $r_{i} d(x, y) \leq d\left(f_{i}(x)\right.$, $\left.f_{i}(y)\right) \leq R_{i} d(x, y)$ for all $x, y \in \mathbb{X}$ and all $i=1,2, \ldots, N$, so that $\mathcal{F}$ is invertible and has a unique attractor $A$. They define the associated fractafold bundle $L$ to be $\left\{\omega \times B_{\omega}: \omega \in \mathbb{I}_{+}\right\}$, but with a certain inductive limit topology in place of the relative topology. They use the projection of $L$ onto $\mathbb{I}_{+}$in order to build a Renault-Deaconu groupoid which exploits the fact that if tails of two strings $\alpha, \beta \in \mathbb{I}_{+}$are the same, and $\mathcal{F}$ obeys the OSC, then $B_{\alpha}$ and $B_{\beta}$ are homeomorphic. This enables the establishment of properties of an associated $C^{*}$-algebra on $L$. The point of view in [11] is very different from that of the present work because it concerns the projection of $L$ onto $\mathbb{I}_{+}$rather than into $\mathbb{X}$.

\section{Acknowledgements}

We thank Alan Carey for interesting discussions and helpful comments. We thank Louisa Barnsley for technical help and suggestions. We thank Krystof Leśniak, who worked with us on the first version of this paper, namely [3], for many comments and suggestions. We thank the anonymous referees for helpful comments. This work was partially supported by a grant from the Simons Foundation (\#322515 to Andrew Vince). It was also partially supported by a grant from the Australian Research Council (\#DP130101738).

\section{References}

[1] Barnsley M.F., Transformations between self-referential sets, Amer. Math. Monthly 116 (2009), 291-304, math.DS/0703398.

[2] Barnsley M.F., Leśniak K., Basic topological structure of fast basins, arXiv:1308.4230.

[3] Barnsley M.F., Leśniak K., Vince A., Symbolic iterated function systems, fast basins and fractal manifolds, arXiv:1308.3819v1.

[4] Barnsley M.F., Vince A., The chaos game on a general iterated function system, Ergodic Theory Dynam. Systems 31 (2011), 1073-1079, arXiv:1005.0322.

[5] Barnsley M.F., Vince A., Developments in fractal geometry, Bull. Math. Sci. 3 (2013), 299-348.

[6] Barnsley M.F., Vince A., Fractal continuation, Constr. Approx. 38 (2013), 311-337, arXiv:1209.6100. 
[7] Barnsley M.F., Vince A., The Conley attractors of an iterated function system, Bull. Aust. Math. Soc. 88 (2013), 267-279, arXiv:1206.6319.

[8] Barnsley M.F., Vince A., Fractal tilings from iterated function systems, Discrete Comput. Geom. 51 (2014), 729-752, arXiv:1310.6344.

[9] Hata M., On the structure of self-similar sets, Japan J. Appl. Math. 2 (1985), 381-414.

[10] Hutchinson J.E., Fractals and self-similarity, Indiana Univ. Math. J. 30 (1981), 713-747.

[11] Ionescu M., Kumjian A., Groupoid actions on fractafolds, SIGMA 10 (2014), 068, 14 pages, arXiv:1311.3880.

[12] Kieninger B., Iterated function systems on compact Hausdorff spaces, Ph.D. Thesis, Augsburg University, Germany, 2002.

[13] Mandelbrot B.B., The fractal geometry of nature, W.H. Freeman and Co., San Francisco, Calif., 1982.

[14] Strichartz R.S., Fractals in the large, Canad. J. Math. 50 (1998), 638-657.

[15] Strichartz R.S., Fractafolds based on the Sierpiński gasket and their spectra, Trans. Amer. Math. Soc. 355 (2003), 4019-4043.

[16] Strichartz R.S., Differential equations on fractals, Princeton University Press, Princeton, NJ, 2006.

[17] Vince A., Möbius iterated function systems, Trans. Amer. Math. Soc. 365 (2013), 491-509. 